\title{
Salvaging an Osseointergrated Implant after Abutment Screw Fracture- A Case Report
}

\author{
Divya Prakash ${ }^{1}$, Paresh V. Gandhi ${ }^{2}$, U. B. Gajare ${ }^{3}$, Prachi B. Bhatia ${ }^{4}$ \\ ${ }^{I}$ (M.D.S.,Deptt. Of Prosthodontics, Bharati Vidyapeeth Deemed University, \\ Dental College and Hospital, Pune) \\ ${ }^{2}$ (Professor, Deptt. Of Prosthodontics, Bharati Vidyapeeth Deemed University, \\ Dental College and Hospital, Pune) \\ ${ }^{3}$ (Professor, Deptt. Of Prosthodontics, Bharati Vidyapeeth Deemed University, \\ Dental College and Hospital, Pune) \\ ${ }^{4}$ (M.D.S.[Final Year], Deptt. Of Prosthodontics, Bharati Vidyapeeth Deemed \\ University, Dental College and Hospital, Pune)
}

\begin{abstract}
The rehabilitation of patients using implants supported prosthesis restores aesthetic and function and preserves the underlying periodontium .Common failure of the implant includes biological and mechanical failure. Biological failures include lack of osseointegration and peri-implantitis. Mechanical failure includes abutment screw loosening or fracture, crown loosening, crown fracture. The most common failure encountered in dental practice is the abutment screw loosening and fracture. Fracture of the implant abutment screw can be a serious problem as the fragment remaining inside the implant may prevent the implant from functioning efficiently. Retrieval of the screw is difficult if the screw fragment is below the neck of the implant and is very small. This case report outlines the management of abutment screw fracture with screw fragment being very small.
\end{abstract}

Keywords : implant, abutment, screw, post, crown

\section{Introduction}

The rehabilitation of patients using implant supported prosthesis restores esthetic and function and preserves the underlying periodontium. The most common failure encountered in dental practice is the abutment screw loosening and fracture. Fracture of the implant abutment screw can be a serious problem as the fragment remaining inside the implant may prevent the implant from functioning efficiently and retrieval is next to impossible with latest screw retrieval kits available today.

Various attempts have been made to retrieve the fractured screw from the implant. The use of an endoexplorer self-made screwdriver and the use of implant repair kit available for some implant systems [1]. However, if the fractured abutment screw fragment is very small, it is difficult to retrieve it and may damage the internal hex of the implant. This case report outlines the management of a fractured abutment screw using a simple technique.

\section{Case Report}

A 40 year old male patient reported to the out-patient section in the Department of Prosthodontics at Bharati Vidyapeeth Dental College and Hospital, Pune, India. The patient complained of loose crowns which were supported by implants (MYRIAD PLUS IMPLANT- D- $3.8 \mathrm{~mm}, \mathrm{~L}-11 \mathrm{~mm}$ ) in the lower right back teeth region. The patient was medically fit and had no other dental complaint.

On intra-oral examination presence of implant supported prosthesis was seen in the 45 and 46 region with mobility of the prosthetic component which was suspected to be abutment screw loosening. The prosthesis of the implant in 45 and 46 were connected by a rigid connector. An intra oral radiograph was made to visualize the problem. Based on radiographic findings it was seen that the abutment was not seating properly on the implant abutment junction as compared to implant abutment with respect to 45. (Fig 1).

An access hole was made from the occlusal surface on 45 and 46 (Fig 2). An attempt was made to tighten the abutment screws. The abutment screw was tightend with respect to 45 . Abutment screw on 46 was removed with the help of hex driver. On examination of the retrieved portion of the screw it was noted that the abutment screw was fractured and only a part of it was retrieved the remainder was present within the implant body. (Fig 3)

Treatment options for management of this condition were as follows:

1. Retriving the screw with screw retrieval kit

2. Fracture of the screw using tungsten carbide bur and removal

3. Fracture of screw with bur and post space preparation in the implant 
4. End of bur or spoon excavator is made into a chisel form and the screw head is engaged and removed. [2]

5. Counter clockwise rotation with a sharp dental explorer or a probe. [3]

It was then decided to create a post space within the internal hex of the implant using a tungsten carbide bur (Endo-Z E0152, DENTSPLY) and air-rotor handpiece. The internal thread and undercuts in the preparation were removed. (Fig $4 \mathrm{a}$ and $4 \mathrm{~b}$ )

Impressions of the post space were made using the indirect technique. Two stage putty reline technique (3M ESPE) was used for making impression of the post space (Fig 5). It was sent to the laboratory for cast post fabrication.

A day after the preparation the patient reported back with severe pain in the 46 region. Probing and radiographic examination revealed a perforation in the implant at the apical third. (Fig 6)

A routine endodontic sealer with epoxy resin (AH Plus, Dentsply) was used to close the perforation and rest of the post space was filled with Zinc oxide Eugenol cement. The patient was recalled the next day for evaluation. As the patient was asymptomatic, the cast post was evaluated for fitting in the mouth and cemented with zinc phosphate cement (Harvard Dental, Germany) and preparation on the core was done to receive the crown (Fig 7). An impression of the prepared core was made with putty light body used previously.

After the coping trial was done (Fig 8) the coping was sent to the lab for final crown fabrication. The crown was luted to the post using zinc oxide eugenol temporary cement (DPI) (Fig 9a and 9b) and the patient was reviewed after 1 week (Fig 10) followed by a one month follow up. The patient has been asymptomatic and is efficiently using the 45,46 region for mastication. Implant protective occlusion was given to the patient. Patient has been recalled after 3 weeks for evaluation.

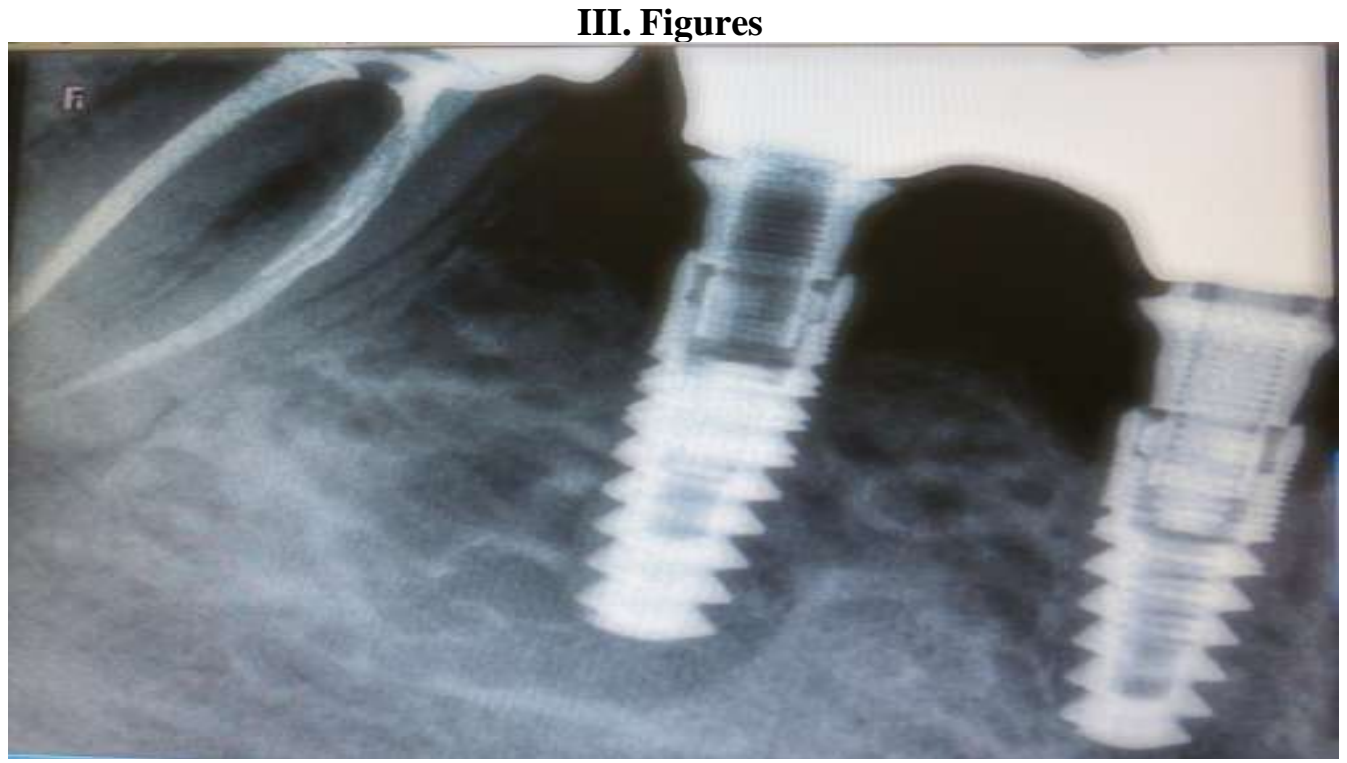

Fig 1: Pre-operative radiograph showing unseating of abutment with 45

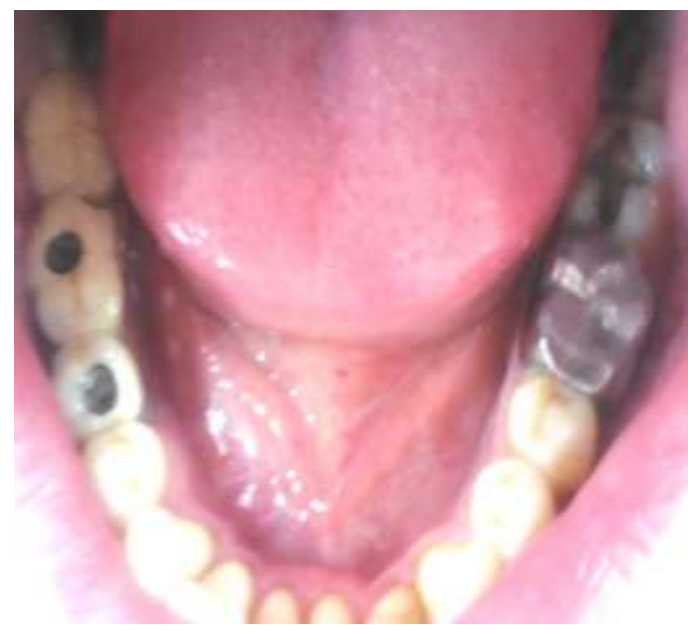

Fig 2: Acess hole made on 45 and 46

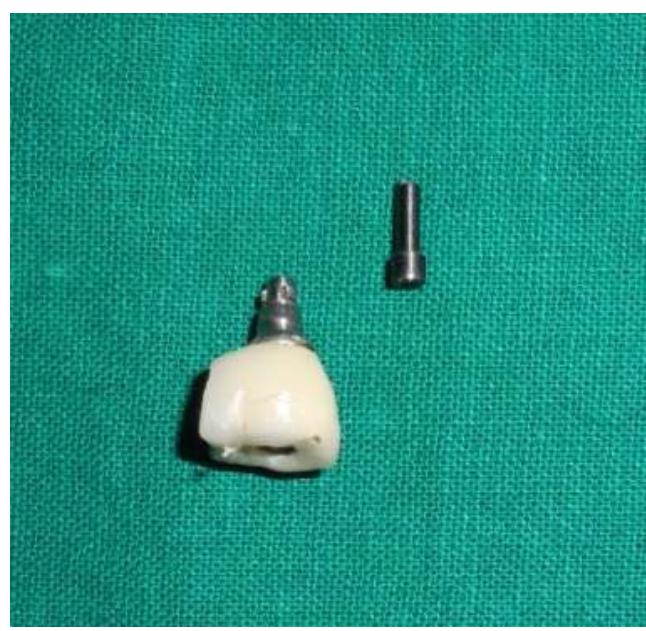

Fig 3: Abutment with fractured screw 


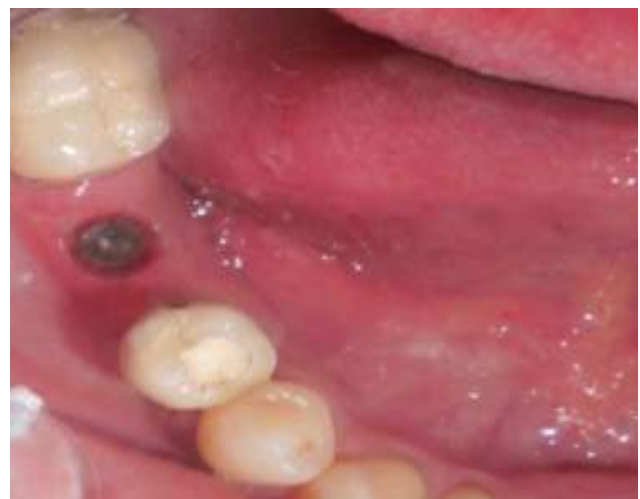

Fig 4(a): Post space preparation

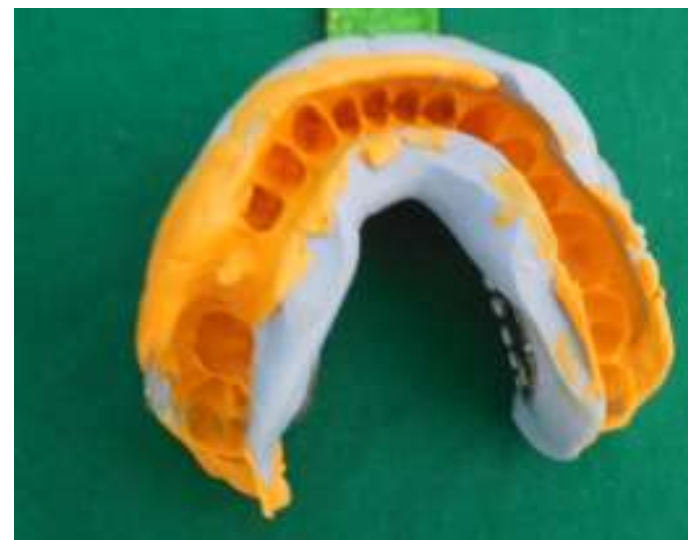

Fig 5: Post space preparation

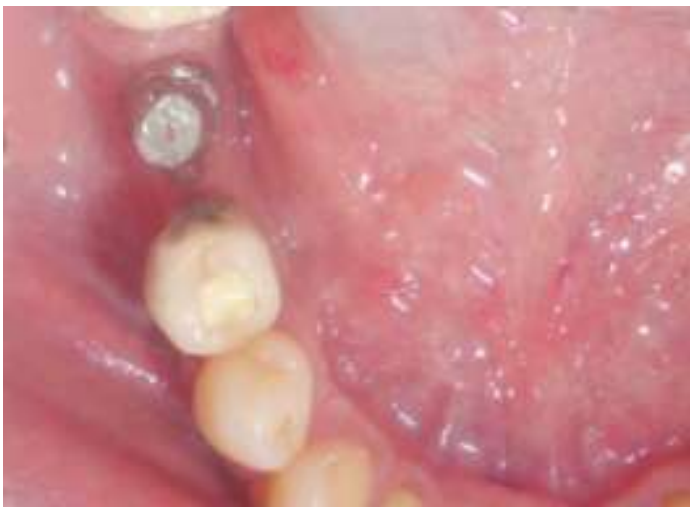

Fig 7: Post Cementation

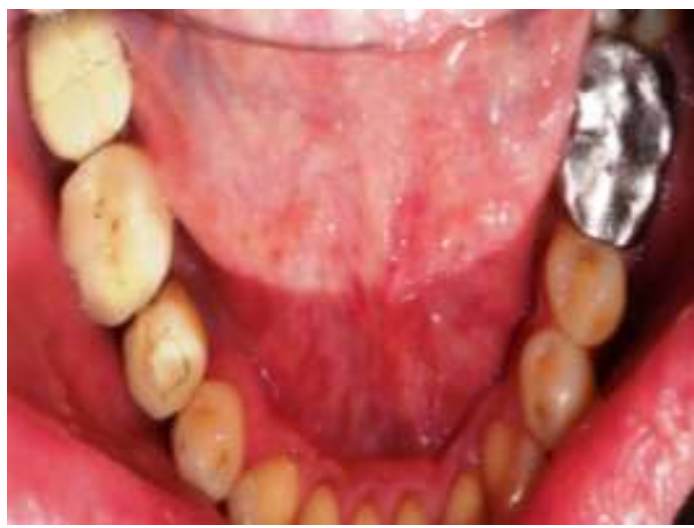

Fig 9(a): Final Prosthesis - Occlusal View

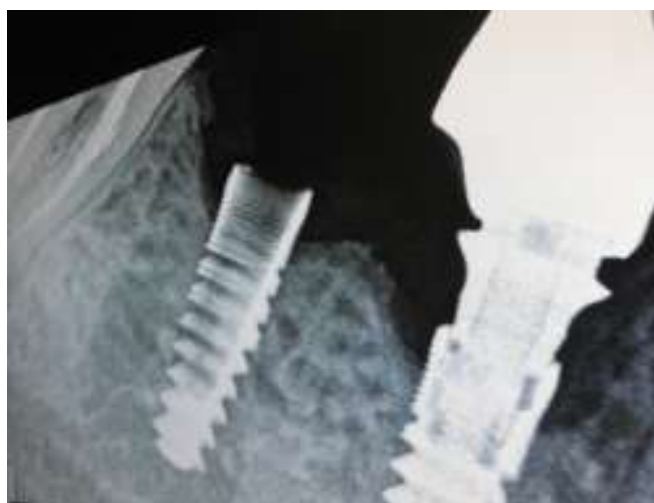

Fig 4(b): Post space preparation -radiographic view

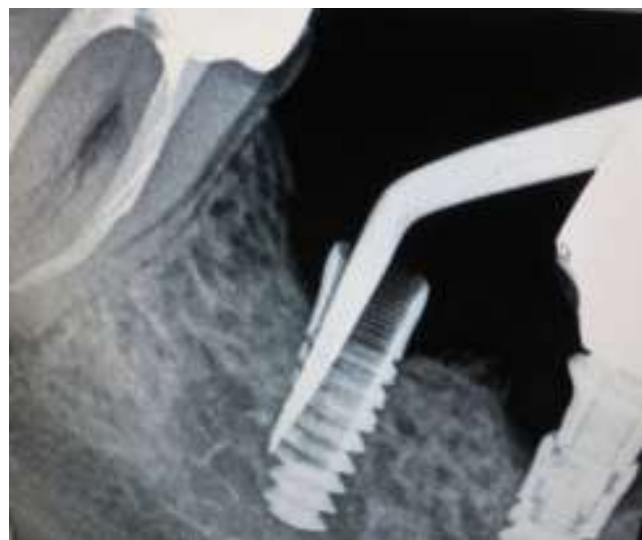

Fig 6: Perforation during preparation

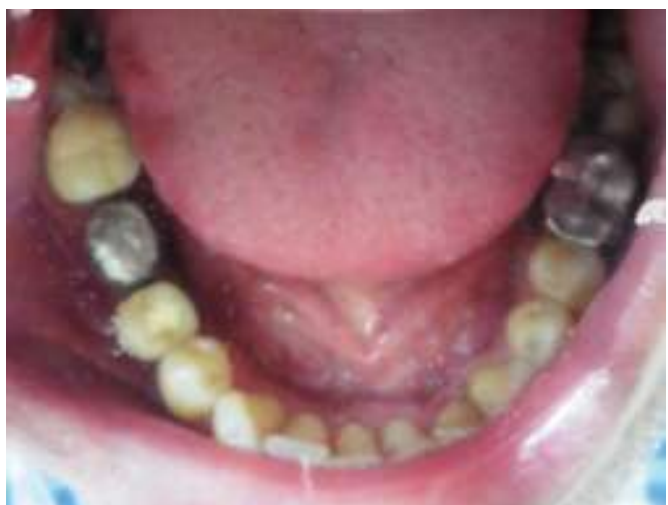

Fig 8: Coping Trial

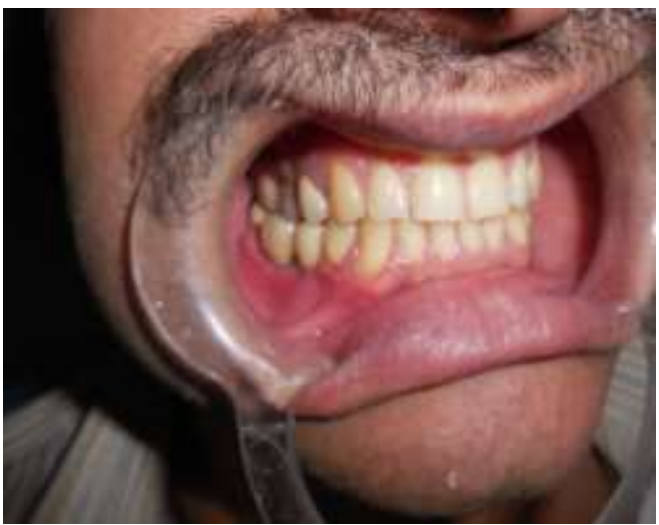

Fig (b): Final Prostheis- In occlusion 


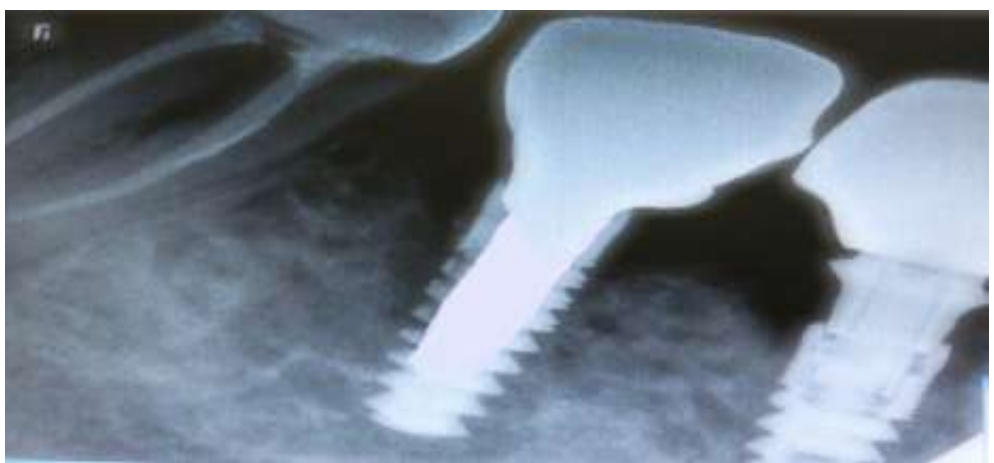

Fig 10: Recall Post Operative radiograph

\section{Discussion}

Implant restorations can fail biologically or mechanically. Biological factors include unsuccessful osseointegration or the presence of peri-implantitis. Mechanical failures include crown fracture, framework fracture, screw loosening, and screw fracture. [4] Causes of screw loosening or fracture are inadequate tightening, adverse occlusal forces, fatigue character and yielding strength of the screw material. [5]

Retrieval with screw retrieval kit and other methods was difficult in this case as the fragment was very small. Secondly fracturing the fragment with bur and removal was not used as this would have damaged the internal threads of the implant and making new prosthesis would have been difficult.

The third treatment option was cost effective and time efficient. Internal wall of the implant is thin so perforation occurred during preparation and risk of perforation is always there. The definitive treatment in this case would have been implant removal but the patient was asymptomatic in subsequent follow up so the implant was not removed.

This technique cannot be used in cases with bone loss as in such cases the height of the post and crown would be excessive and it will act as a vertical cantilever.

The longevity of the restoration is also questionable as the post is retained with cement compared to the screw retained abutment. It will depend on the maintenance by the patient as well as regular recall visit by the dentist.

\section{Conclusion}

The aim of prosthetic rehabilitation with implants should be to prevent fracture of implant components and technique like above should be used exceptionally. This case was an attempt to salvage an ailing implant and would require follow up.

\section{References}

[1]. Walia S.M., Arora S, Luthra R, Walia K.P. Removal of Fractured Dental Implant Screw Using a New Technique: A Case Report. Jounal of Oral Implantology $2012 ; 38: 747-750$.

[2]. Satwalekar P, Chander KS, Reddy BA, Sandeep N, Sandeep N, Satwalekar T. A Simple and Cost Effective Method used for Removal of a Fractured Implant Abutment Screw: A Case Report. J Int Oral Health 2013; 5(5):120-3.

[3]. http://www.oralhealthgroup.com/features/retrieval-of-fractured-prosthetic-screws-from-dental-implants-a-case-report

[4]. Harshakumar K, Bhatia S, Ravichandran R, Joy PT. Salvaging an implant with abutment screw fracture by a custom titanium post and core supported prosthesis - A novel technique. International journal of scientific study. April 2014; 2: 36-39.

[5]. Satterthwaite J. , Rickman L. Retrieval of fractured abutment thread from an implant : A case report. British Dental Journal. 2008; 204: $177-180$ 